\title{
Regards historiques sur le fait maritime
}

Un essai de comparaison franco-russe

\section{Sylviane Llinares, Alexei Kraikovski et Irina Gouzévitch}

\section{(2) OpenEdition}

Journals

Édition électronique

URL : https://journals.openedition.org/artefact/9728

DOI : $10.4000 /$ artefact. 9728

ISSN : 2606-9245

Éditeur :

Association Artefact. Techniques histoire et sciences humaines, Presses universitaires du Midi

\section{Édition imprimée}

Date de publication : 7 octobre 2021

Pagination : 9-34

ISBN : 978-2-8107-0755-3

ISSN : 2273-0753

\section{Référence électronique}

Sylviane Llinares, Alexei Kraikovski et Irina Gouzévitch, « Regards historiques sur le fait maritime », Artefact [En ligne], 14 | 2021, mis en ligne le 25 août 2021, consulté le 03 septembre 2021. URL : http:// journals.openedition.org/artefact/9728; DOI : https://doi.org/10.4000/artefact.9728

\section{(c) (†) $\ominus$}

Artefact, Techniques, histoire et sciences humaines est mise à disposition selon les termes de la Licence Creative Commons Attribution - Pas d'Utilisation Commerciale - Pas de Modification 4.0 International. 


\section{Regards historiques sur le fait maritime}

\section{Un essai de comparaison franco-russe}

\section{Sylviane Llinares, Alexei Kraikovski et Irina Gouzévitch}

$\mathrm{L}$

es aspects maritimes de l'histoire russe et française semblent difficiles à comparer au premier regard : trop différentes sont les voies empruntées par l'un et l'autre des deux pays. Cependant, à la fin du $\mathrm{xx}^{e}$ et au début du $\mathrm{xxI}^{\mathrm{e}}$ siècle, les nouvelles approches de l'histoire maritime ont créé un terrain fertile à une étude croisée de la maritimité entre la France et la Russie. Selon Ann Day et Ken Lunn, la représentation même de ce que signifie le mot «maritime » appliqué à l'histoire et au patrimoine, a dû être repensée ${ }^{1}$. Des tentatives réitérées plus ou moins vigoureuses ont remis en question le paradigme traditionnel de l'histoire maritime où la composante militaire avait jusqu'alors prévalu.

On peut dégager quelques tendances générales qui déterminent aujourd'hui le développement des études maritimes. La première est caractérisée par une attention accrue à l'importance de la nature maritime et océanique en tant qu'entité active qui possède son " agency " propre, à savoir une capacité inhérente d'interagir avec la société humaine. Cette approche constitue le fondement du « tournant océanique » dans les sciences humaines. Ce dernier concept est décrit comme une remise en question de la somme des interactions expérimentées par la société dans son rapport avec l'élément marin ${ }^{2}$. Locéan est ici considéré comme une force active qui interfere avec

1. Day et Lunn, 2003, p. 289-305.

2. DeLoughrey, 2017, p. 32.

95 Sylviane Llinares, Alexei Kraikovski et Irina Gouzévitch, « Regards historiques sur le fait maritime. Un essai de comparaison franco-russe », Artefact, 14, 2021, p. 9-34. 
l'homme de plusieurs façons différentes. Ses caractéristiques naturelles, y compris le relief sous-marin, l'état des organismes qui le peuplent, la composition chimique de l'eau et d'autres caractéristiques sont prises en compte lors de l'analyse de l'expérience maritime de l'humanité. Ainsi, la mer n'est plus perçue comme un objet passif sous l'action de l'homme. Ses particularités d'élément puissant et redoutable sont reconnues comme substantielles par l'historien ${ }^{3}$.

La deuxième tendance tient au fait qu'une telle évolution de l'histoire maritime relève moins de l'essor des directions traditionnelles de recherche telles que l'histoire navale ou l'histoire du commerce maritime, que de l'approche intégrée et aussi globale que possible du sujet d'étude. Comprise ainsi, l'histoire maritime se veut transfrontalière, recelant un énorme potentiel d'études comparatives et s'ouvrant sur des sujets connexes, telle l'histoire des zones côtières qui s'étendent parfois très loin de la mer ${ }^{4}$.

Il existe également une approche synthétique issue de la fusion des deux précédentes; elle consiste à aborder l'histoire des océans et des mers comme une unité naturelle et culturelle. Cette direction historiographique, qui remonte à la tradition fondée par Fernand Braudel à travers ses travaux sur le monde méditerranéen ${ }^{5}$, s'est développée dans le cadre des études des différents mondes océaniques : atlantique, pacifique et celui de l'Océan indien ${ }^{6}$. Parmi les grands classiques en matière d'histoire des mers, citons aussi l'ouvrage de Michael North consacré à l'histoire de la Baltique ${ }^{7}$. Le travail visant à penser l'histoire maritime comme un processus d'échelle globale, a culminé dans la publication de l'ouvrage collectif en plusieurs volumes $\mathrm{La} \mathrm{Mer}$ dans l'histoire, fruit de la coopération d'historiens reconnus à l'international ${ }^{8}$.

Enfin, la troisième tendance qui gagne en importance consiste à interroger le phénomène même de la maritimité (maritimeness, maritimity) comme un ensemble de caractéristiques spécifiques qui rendent telle ou telle communauté « maritime ». Une définition sans équivoque du terme maritimité

3. Miller (dir.), 2013.

4. Harlaftis, 2010, p. 211-229.

5. Braudel, 1966.

6. Greene et Morgan (dir.), 2009 ; Armitage et Bashford (dir.), 2014 ; Alpers, 2014.

7. North, 2015.

8. Buchet, Arnaud et De Souza (dir.), 2017 ; Buchet et Balard (dir.), 2017 ; Buchet et Le Bouëdec (dir.), 2017 ; Buchet et Rodger (dir.), 2017. 
n'a pas encore été développée, l'élaboration de ce concept étant avant-gardiste dans le domaine de l'histoire marine et côtière. Ainsi, l'anthropologue Jeffrey Fleisher propose la description d'une communauté maritime à travers un système d'influences mutuelles. Il considère qu'une communauté ne peut être appelée maritime que lorsque la nature marine adjacente à son habitat a un impact apparent et qu'elle est elle-même influencée par un large éventail d'organisations, de pratiques et de croyances socioculturelles liées à la mer ${ }^{9}$. Pour le sociologue Yarin Eski, la maritimité peut être décrite comme une qualité spécifique de l'infrastructure d'exploitation marine qui détermine l'identité des personnes qui y sont liées, par exemple, les employés de la police portuaire ${ }^{10}$. Enfin, dans le domaine des études du patrimoine, la maritimité est mise à la même échelle que les concepts de culture marine et de relation entre l'homme et la mer, conditionnés par une valeur culturelle, émotionnelle ou pratique ${ }^{11}$.

La polysémie du terme maritimité a été détaillée par Carlotta Andrews dans ses travaux consacrés à l'étude du patrimoine maritime des Bermudes ${ }^{12}$. Ayant noté que la maritimité, à savoir le lien avec la mer, est devenue la base de l'identité locale, la chercheuse a décrit le patrimoine maritime des Bermudes comme une résultante de cinq dimensions : $1^{\circ}$ l'interaction entre le passé et le présent maritimes à travers la mémoire, la nostalgie et l'expérience en direct ; $2^{\circ}$ l'oubli et la résurgence du passé maritime ; $3^{\circ}$ le fait de cultiver des identités sous-tendues par les relations affectives avec les archétypes maritimes; $4^{\circ}$ la construction de représentations de la maritimité et des identités qui y sont attachées ; $5^{\circ}$ la réparation du tissu communautaire et la lutte active contre l'exclusion sociale par le recours à la maritimité.

\section{La maritimité en Europe}

Le concept de maritimité apparu au début des années 1990 et emprunté aux géographes désigne donc les relations entre les sociétés et la mer et les différentes façons de s'approprier la mer. L'étude de ces relations inclut la question des représentations collectives et les perceptions portées par les

9. Fleisher et al., 2015.

10. Eski, 2016.

11. Alegret et Carbonell Camós (dir.), 2014.

12. Andrews, 2010. 
groupes sociaux. Si elles nourrissent un imaginaire puissant, ces représentations sont aussi prescriptives, elles ont une valeur pratique en proposant des manières de se projeter sur les ressources que peut offrir la mer ou les plaisirs qu'elle peut procurer à mesure que s'affirme le désir du rivage ${ }^{13}$. Au XVIII ${ }^{\mathrm{e}}$ siècle, Louis-Sébastien Mercier moque les Parisiens qui doivent gagner le littoral normand pour se régaler de poisson frais :

Le poisson de mer n'est pas à bon marché à Paris, malgré quelque diminution sur les entrées, soulagement dû à $\mathrm{M}$. Turgot. Il n'est presque jamais frais. Il ne peut venir que des côtes de Normandie ou de Picardie, le poisson non salé ne pouvant souffrir le transport au-delà de trente à quarante lieues. Les approvisionnements de la Cour enlèvent tout ce qu'il y a de plus beau, $\&$ le Parisien mange le fretin. Notez que les Chartreux, les Carmes, les Bénédictins, les Minimes, \& les autres Religieux qui font maigre, affament la ville de poisson, \& entretiennent la cherté, en payant fort cher tout ce qui est à leur convenance.

Les entrées du poisson nuisent à l'impôt, parce qu'il n'est pas assez modéré. Le Parisien qui veut se régaler de marée, est obligé de se transporter à Dieppe ; $\&$ le Bourgeois, quand il devient un peu cossu, fait d'abord ce voyage-là tout seul ; ensuite il y mène sa ronde femme. Ils restent en extase devant l'Océan, \& ils n'ont pas tort ; mais ils croient avoir touché les colonnes d'Hercule, $\&$ se hâtent de rentrer dans leurs foyers. Ils sont si transportés, si enchantés de leur voyage, que le reste de leur vie ils en parleront tous les soirs à leur souper devant leurs filles $\&$ la servante ébahie ${ }^{14}$.

On validera également le concept en observant les arts visuels dans toutes leurs registres, académiques ou populaires, tels les grands ports de France peints par Joseph Vernet ou les gravures d'après les dessins de Nicolas Ozanne $^{15}$. Les savoir-faire et les techniques entrent dans le champ de la maritimité qui peut, de plus, ouvrir sur les futurs d'anticipation. Enfin, la maritimité renvoie à la notion d'héritage, de mémoire collective et de patrimoine. La maritimité n'est donc ni figée, ni déterminée, son sens varie selon les époques, les idéologies, l'évolution des techniques et des

13. Corbin, 1990.

14. Mercier, 1783 , t. 1 , p. 60-61.

15. Llinares, 2011. 
mentalités. Ainsi a-t-on analysé l'émergence d'une nouvelle maritimité à partir des années 1970 en France lorsque les formes traditionnelles entrèrent en crise (construction navale, pêche) et furent remplacées par de nouvelles pratiques sociales liées à la reconversion de friches portuaires, au tourisme maritime et à la croissance des sports nautiques ${ }^{16}$.

La maritimisation du monde est un autre concept permettant d'interroger la relation entre mer et histoire. Le phénomène de maritimisation a donné lieu à un renouvellement des approches historiques afin d'en déceler plus précisément les mécanismes. La mer offre, au fil des siècles, un point de vue unique sur l'accélération des processus de développement. L'exploitation croissante des ressources océaniques et la transformation accrue des littoraux par l'homme, les navigations de plus en plus denses, qui assurent la majeure part des échanges autour des points névralgiques que sont les ports ou bien encore la régulation et le contrôle des espaces maritimes, ainsi que les missions, toujours plus nombreuses, assurées par les flottes nationales font de la mer l'enjeu et le miroir menacé des transformations du monde ${ }^{17}$. La maritimisation ne se résume pas à la question des transports maritimes. Les mers et les océans font figure de « dernière frontière " du monde contemporain, ce qui confère à la maritimisation une dimension géopolitique et géostratégique. L'importance du processus global de maritimisation n'exclut d'ailleurs pas la " démaritimisation " de certains lieux. Ainsi, André Vigarié, fondateur de la géographie portuaire et maritime française, l'a-t-il proposée pour des villes-ports en Europe du Nord-Ouest, liée à la diversification de leur économie. Les États sont confrontés à la redéfinition géopolitique des océans. Dans un rapport de 2012, fait au nom de la Commission des Affaires étrangères, de la Défense et des Forces armées, il est question des enjeux de la maritimisation pour la France, entre menaces et opportunités, tels que la liberté de circulation en mer, l'épuisement des ressources, la territorialisation des espaces maritimes, qui entrainent l'émergence de rivalités nouvelles entre grands pôles de développement ${ }^{18}$. Les réponses à de tels défis impliquent un savoir-faire et une maîtrise technologique en matière de construction navale, d'exploration et d'exploitation en haute mer de premier plan, ainsi qu'une

16. Péron et Rieucau (dir.), 1996.

17. Coll, 2016.

18. Lorgeoux et Trillard, 2012. 
marine de guerre puissante en capacité de défendre le deuxième territoire maritime du monde ${ }^{19}$.

Qu'en est-il dans le passé ? Au XviII ${ }^{e}$ siècle, "l'expérience de la mer ", en Europe, selon l'expression de Liliane Hilaire-Pérez ${ }^{20}$, ouvre sur la question d'un monde transformé par la globalité dont les contemporains ont eu conscience, comme l'écrit Antoine Lilti à propos des grands défis des Lumières $^{21}$. Qu'il s'agisse d'économie politique ou d'histoire, les auteurs et penseurs partagent alors le constat d'un monde bouleversé depuis le $\mathrm{XVI}^{\mathrm{e}}$ siècle par le développement du grand commerce maritime, les rivalités impériales, la circulation des biens et des idées. Cette interdépendance du monde oblige à repenser la mise en ouvre des politiques maritimes et invite l'historien à reconsidérer l'histoire politique du " fait maritime ". Maîtrise du littoral, projection de puissance, contrôle des populations, perception des enjeux économiques, exploration des océans et incitation au développement des activités maritimes caractérisent les champs d'action de l'État dans la longue durée. Il s'agit de comprendre les rôles que l'on assigne à l'espace maritime, et nous dirions que la problématique la plus prégnante est celle de la redéfinition permanente de la gestion de cet espace en fonction des besoins. L'historien en changeant ses points de vue subit aussi l'influence de son contexte, celui des grands défis environnementaux $\mathrm{du} \mathrm{XxI}^{\mathrm{e}}$ siècle et de la dernière phase de la mondialisation des échanges. Parmi toutes les activités liées à l'action de l'État en mer, celle de la sauvegarde de l'environnement marin accompagne désormais les missions plus traditionnelles de la sûreté du transport maritime.

Si l'on se réfère aux interrogations portées sur la validité même du concept d'histoire maritime (plus récemment dénoncée comme une construction historiographique européocentrée), il est légitime aussi de s'interroger sur la définition de ce que peut être une politique dite maritime au XVIII ${ }^{\mathrm{e}}$ siècle. Cette définition présuppose la mobilisation de plusieurs champs de recherche et renvoie finalement à toute une série de regards

19. Le domaine maritime français est le deuxième du monde juste derrière les États-Unis, dans la zone des 200 milles nautiques $(365 \mathrm{~km})$ à partir de la côte, l'État riverain peut exploiter les ressources économiques (pêche, exploration et exploitation pétrolière et minière). Les pays ont aujourd'hui la possibilité d'étendre leur zone exclusive jusqu'à 350 milles nautiques soit $650 \mathrm{~km}$ (seulement si elle fait partie du plateau continental).

20. Hilaire-Pérez, 1997.

21. Lilti, 2019. 
historiques portés sur le "fait maritime ». Celle-ci présente un défi à l'historien par la complexité des sources et des méthodes qui doivent entrer en jeu et implique la mise en ouvre d'une transdisciplinarité pour mieux comprendre l'interaction entre des systèmes environnementaux, économiques, sociaux et culturels.

Questionner l'action mûrie au centre du pouvoir et les processus de changement (ou non) de l'utilisation de la mer et du littoral au cours du $\mathrm{XVIII}^{\mathrm{e}}$ siècle renvoie l'historien à sa propre temporalité dans la perception des problèmes, sans que ceux du présent ne brouillent nécessairement l'analyse du passé.

\section{Des outils conceptuels de mise en comparaison des maritimités}

Il convient, d'abord, d'évoquer le concept d'imaginaire (imaginaries). Ainsi, Sam Robinson considérait l'océan comme un élément important des "imaginaires sociotechniques" (sociotechnical imaginaries). Notant que le processus de prise de décision en matière d'évolution de la société " est souvent fondé sur des scénarios et des technologies purement imaginaires », il soulignait qu'il fallait savoir distinguer " l'imagination d'une simple fantaisie ou illusion » de l'imagination comme " ressource culturelle communautaire qui stimule l'activité sociale et politique ». En se fondant sur les matériaux diplomatiques du milieu du $\mathrm{xx}^{\mathrm{e}}$ siècle, il a montré que "l'imaginaire sociotechnique » d'une part, était déterminé par les représentations générales sur les propriétés de l'océan et les normes de son interaction avec la société humaine et, d'autre part, que cet imaginaire a lui-même façonné de nombreuses décisions et actions réelles ${ }^{22}$. Cette approche de l'imaginaire sociotechnique permet de voir d'un œil nouveau les prises de décision ou les pratiques sociales, tandis que le " tournant océanique " a souligné le rôle des conditions naturelles dans la formation de l'imaginaire.

Il est possible de poursuivre la réflexion sur les imaginaires en abordant la question des circulations de savoirs et du rôle de la traduction, entendue de manière extensive, incluant l'expérience visuelle. L'idée de la traduction visuelle relève largement encore de l'outillage des historiens de l'art et n'a 
été appliquée que parcimonieusement à l'histoire maritime, principalement à propos des contacts maritimes lointains, comme base des emprunts en matière d'art figuratif ${ }^{23}$. Dans un autre domaine, l'architecture, Esra Akcan a décrit le lien profond entre la traduction visuelle et la prise de décision dans les domaines politique, économique et social. De plus, elle a montré que les résultats de la traduction visuelle diffèrent assez nettement de l'original, même s'ils en sont une copie exacte au sens technique du terme, précisément en raison de la différence "imaginaire " qui détermine l'existence sociale de l'original et de la traduction ${ }^{24}$. Ce cadre théorique nous paraît prometteur pour aborder sous un angle nouveau la question du transfert de l'expérience maritime européenne entre la France et la Russie.

\section{L'expérience maritime russe}

Malgré le nombre imposant de travaux et l'état élaboré de certains sujets, l'héritage historiographique russe se compose en grande partie des récits disparates (l'histoire navale, l'histoire des découvertes et des explorations) inscrits dans le paradigme de l'histoire des politiques publiques passablement éloigné de l'histoire mondiale de l'appropriation des océans et de leurs littoraux ${ }^{25}$. Si la place de la mer et du littoral dans l'histoire de l'Europe est devenue un objet d'étude et d'interprétation dans le cadre d'un grand projet international ${ }^{26}$, pour la Russie une telle question ne s'est même pas posée. Le renouvellement historiographique ouvre donc la voie à une histoire de la maritimité entre la France et la Russie. De plus, cette nouvelle perception du fait maritime, riche et complexe, est tout-à-fait adaptée à l'analyse comparée de la dimension maritime des deux pays. Leur appartenance commune à l'histoire européenne en est le gage.

L'union entre la mer et l'Europe qui caractérise les temps modernes est un fait majeur, bien perçu par les contemporains, tel un arcane de la puissance et de la modernité, suffisamment comprise et analysée pour que se développent des politiques maritimes à grande échelle. La mer est d'abord une expérience ${ }^{27}$, collective et européenne, qui révèle une diversification

23. Le Corbeiller, 1968.

24. Akcan, 2012.

25. Leikin, 2016.

26. François et Isaacs (dir.), 2001.

27. Hilaire-Pérez, 1997. 
des vécus maritimes à une échelle multiscalaire marquée pat un jeu inégal entre les acteurs et des ambitions concurrentielles.

La France moderne offre certainement un bon exemple de l'expérience européenne précoce d'interaction avec la mer. La Russie avant le XVIII ${ }^{\mathrm{e}}$ siècle présente un profil inverse : loin d'être perçue comme un élément de l'identité nationale, la maritimité est le lot des petites communautés côtières. En empruntant pour notre compte la terminologie utilisée dans la « Nouvelle histoire côtière ", on peut dire que pour la Russie, l'interaction avec la mer faisait partie de l'expérience côtière plutôt que maritime. Dans ce domaine de recherche, l'ensemble complexe d'aspects de mise en valeur des zones côtières (couvrant à la fois le littoral et la mer côtière) est pensée à travers le concept d' "expérience côtière " (coastal experience, littoral experience). Le terme fait référence aux modes de vie et d'activités multiformes que la société a accumulés au fil des siècles et même des millénaires dans le traitement de la nature côtière, et qui permettent de développer des stratégies optimales d'adaptation aux conditions tout en tirant profit de leur exploitation $^{28}$. Dans le même temps, l'expérience russe avait une spécificité, celle de faire de la " maritimisation » un élément de l'« européanisation ». Le rôle attribué à la mer dans les réformes pétroviennes au premier quart du XVIII $^{\mathrm{e}}$ siècle ne fait aucun doute. Ce lien semble même si bien admis que le phénomène de la "maritimisation » de la Russie n'a jamais été vraiment questionné ni pensé dans sa complexité. Il s'agissait principalement d'examiner les efforts de l'État pour créer une marine de guerre, et cette optique navale a conduit à une vision plutôt déséquilibrée du développement des relations de la Russie avec la mer. Ainsi, Pavel Krotov, en essayant de comprendre les relations " incertaines » de la Russie avec la mer a mis l'accent, comme de coutume, sur l'aspect naval de la politique de l'État et a conclu que

la création de la marine russe de Baltique ne suit pas le schéma traditionnel : on a d'abord développé la pêche, puis le commerce, et ensuite la création de la marine pour protéger les intérêts de l'État en mer. Pierre le Grand, au contraire, a engagé la transformation de la Russie en une grande puissance maritime avec la construction de navires militaires. Puis il a commencé une campagne pour attirer les navires marchands étrangers dans les ports russes. Ce n'est

28. Worthington, 2017, p. 5. 
qu'à l'issue de cette campagne que des mesures ont été prises pour développer la navigation marchande avec des navires russes. L'État n'était pas intéressé par la pêche ${ }^{29}$.

Mais le concept de maritimité implique inévitablement la nécessité d'une mise en perspective plus large. En effet, si l'expérience maritime de l'Europe était vaste et diversifiée, alors son emprunt par la Russie dépassait probablement le cadre des mesures prises par l'État pour construire et utiliser efficacement les navires de guerre. Ainsi, la question majeure des recherches sur la "maritimisation de la Russie " revient à savoir comment le transfert des expériences, des connaissances et des techniques a eu lieu. Si la maritimité détermine l'existence des liens avec la mer dans une communauté particulière, alors comment un observateur externe peut-il déterminer que cette communauté est maritime ? Comment peut-il dégager les composants de la maritimité ? Enfin, comment ces particularités de la vie publique et sociale peuvent-elles être reproduites dans une autre société afin d'en faire, à son tour, une société maritime ? Il semble que ce soit une tâche non seulement pour l'histoire maritime russe, mais plus généralement, une mission pour l'avenir pour comprendre l'expérience maritime dans son ensemble. À ce stade, il est possible de définir un certain nombre d'approches théoriques qui fournissent des outils d'analyse pratiques et efficaces.

\section{Jeux d'échelles et perceptions}

Dans un ouvrage récent sur l'écriture de l'histoire globale, Christian Grataloup s'interroge sur le pluriel des sociétés qui se monnaie « en autant de récits créant ainsi une carte des histoires ». Il emploie l'expression de "géographie des temporalités » qui elle-même a sa propre histoire ${ }^{30}$. Pour cela, dit-il, il faut commencer " par jauger la pertinence des outils dont on dispose ". Comment penser les autres sociétés, si ce n'est avec sa propre boîte à outils ? On comprend parfaitement l'enjeu dans l'écriture d'une histoire du monde, destiné à modérer tant l'impérialisme que le relativisme intellectuel. Christian Grataloup explique qu'il existe une troisième voie : " en changeant de niveau dans l'échelle des sociétés, on gagne en extension, tout le problème est de ne pas trop perdre simultanément en

29. Krotov, 2016. p. 522.

30. Grataloup, 2011. 
approfondissement ». Définir sa boite à outils et le jeu des échelles que l'on retient est évidemment nécessaire à toute recherche.

L'on pourrait appliquer cette idée du changement des focales à l'histoire maritime et littorale française. Celle-ci a fait l'objet de travaux d'envergure depuis un bon demi-siècle, en témoigne le bilan édité en 2010 accompagné d'une bibliographie de plus de 2200 références $^{31}$. Dans l'introduction, Gérard Le Bouëdec rappelait la formation du Groupement d'intérêt scientifique français en histoire maritime (2005) et le consensus qui s'était fait autour de quelques points essentiels : « les usages de la mer et les rapports pluriels de l'homme au littoral qui constituent le socle conceptuel de l'histoire maritime. À quelles échelles ?32 "Cette question des échelles s'applique aux espaces littoraux, qui sont des espaces d'interface pour les sociétés en contact avec les mers, elle s'applique à ces contrées dans la mesure où "l'appel de la mer" a une incidence sur la politique, l'économie, la société et les pratiques culturelles. Elle s'applique enfin à un niveau global qui envisage le rôle de la mer dans le développement d'un pays ou d'un continent. De fait, avec une approche aussi vaste, la question de la politique maritime suivie par un État et ce qui la caractérise doit permettre de travailler à tous les niveaux. L'historiographie française est riche de ce point de vue, mais elle a souvent « livré une histoire segmentée des façades océaniques » et comme l'explique Gérard Le Bouëdec, il restait à construire une histoire des flux intermédiaires, celle des modestes chantiers, du cabotage, des petites pêches, des populations littorales.

Au vu de toute la production disponible, force est pourtant de constater que l'on ne dispose pas d'une histoire "totale " du fait maritime en France $^{33}$. Elle est sans doute la plus difficile à mener ; selon les sensibilités épistémologiques, tel ou tel aspect sera privilégié, de plus la partition entre le commerce et la guerre a un peu estompé la visibilité du global et donc de l'action des pouvoirs. D’où la nécessité de projeter de nouvelles études pour écrire, en France, une histoire plus politique de l'économie maritime. Les conditions de son développement ont d'ailleurs été présentées dans la grande et belle synthèse Les Français, la terre et la mer ${ }^{34}$. Dans l'introduction, les auteurs ont insisté sur « une conception complexe du rapport de

31. Le Bouëdec, 2010.

32. Le Bouëdec, 2010, p. 8.

33. Villain-Gandossi et Rieth (dir.), 2001.

34. Cabantous, Lespagnol et Péron (dir.), 2005. 
la France à la mer, recouvrant une multiplicité de relations sur différents plans ", en définissant comme premier type de relations, celle d'ordre politique « en termes de pouvoir, de contrôle, de puissance ». Cependant, dans cette approche du rapport de l'État à la mer, il semble que l'on adopte encore des schémas chrono-antagoniques sur la contribution de la monarchie bourbonienne à l'activité maritime. On oppose volontiers un $\mathrm{XVII}^{\mathrm{e}}$ siècle rayonnant qui a tout pensé, tout construit et tout organisé, non sans résistance, à un XVIII ${ }^{\mathrm{e}}$ siècle, un peu plus pâle en termes d'innovations institutionnelles, "sous la contrainte " pour la gestion des littoraux et des hommes, et où le commerce maritime (grand et petit) fonctionne presque de manière autonome sur fond de croissance économique éblouissante, les périodes de guerre obérant pour un temps ces beaux résultats ${ }^{35}$. Comme si le destin des deux flottes, commerciale et militaire, n'était pas lié, comme si l'une, la commerciale, compensait la faiblesse numérique de l'autre, du moins jusqu'aux années 1780. Même lorsqu'il s'agit du domaine naval, on lit parfois des critiques assez sévères sur le règne de Louis XVI, cela malgré la guerre d'Amérique et la force retrouvée de la Marine ${ }^{36}$. Ces perspectives correctrices renvoient à la lecture que l'on pouvait faire d'une " histoire maritime française " au temps de la Restauration. Assez édifiante est par exemple l'évocation historique faite dans l'ouvrage Le parfait capitaine par Laget de Podio, ancien procureur du roi et avocat à Marseille. Dans son introduction, il explique les conditions de la puissance commerciale maritime d'une nation et met logiquement en évidence l'apport français au droit maritime - la grande ordonnance de Marine de 1681 - définissant des temps forts, tout en négligeant le XVIII ${ }^{\mathrm{e}}$ siècle $^{37}$.

Enfin, dernier point négatif, et non des moindres, la France est souvent présentée comme un pays de terriens, qui n'entend que par intermittence l'appel de la mer, un "pays allergique " pour reprendre la formule de Christian Buchet ${ }^{38}$. C'est bien souvent un argument commode pour expliquer une histoire qui n'a rien de linéaire, qui n'est pas spécifique non plus, et surtout des choix parfois très défavorables au secteur maritime pris dans toutes ses acceptions. Peut-être, associe-t-on trop souvent en les recouvrant parfaitement, politique maritime et puissance maritime.

35. Petre-Grenouilleau, 1997.

36. Vergé-Franceschi, 1998.

37. Laget de Podio, 1828 et 1835.

38. Buchet, 2004, p. 186. 


\section{Politique maritime et puissance maritime}

Étienne Taillemite (1924-2011) déplorait que la puissance maritime fût absente des mentalités françaises :

La France n'a guère cessé de méconnaître le rôle de la puissance maritime dans la vie des peuples, aussi bien dans ses aspects militaires que dans ses fonctions économiques et culturelles, celle-ci étant capitale en raison des possibilités infinies qu'offre la mer comme facteur d'ouverture sur le monde et de contacts entre civilisations différentes ${ }^{39}$.

Mais on le voit, le débat se situe d'abord sur la contestation périodique de la nécessité de posséder une marine de guerre et le faible intérêt porté par l'opinion publique et la classe politique aux questions maritimes. Il semble qu'il y ait un ennemi intérieur de la puissance maritime en France et que la classe politique, notamment à l'époque contemporaine, y ait sa part de responsabilité. C'est donc une histoire en creux qui propose de s'intéresser aux discontinuités de l'effort maritime du pays, faisant de la marine d'État la grande victime. Si l'on admet, comme Étienne Taillemite que ce courant négatif fut refoulé pour un temps par la guerre d'Indépendance américaine (1776-1783), alors il est d'autant plus intéressant d'analyser la politique maritime à cette époque car elle ne saurait se limiter aux seules opérations navales. Le vrai défi est sans doute celui de parvenir à mettre tous les acteurs en action dans une vision panoramique, voire systémique. Définir la politique maritime de la France suppose que l'on relie différentes histoires, que l'on essaie de clarifier la sémantique des domaines qui l'animent, et il faut nécessairement le faire à partir de l'économie maritime à l'époque moderne.

A contrario, l'analyse que propose Nicolas Rodger sur les fondements qui permettent de parvenir à la puissance maritime mérite d'être rappelée. Les sociétés ouvertes et pluralistes conviennent mieux, dit-il, non seulement pour innerver le soutien politique dont la Marine a besoin, mais aussi pour édifier une puissante marine. La compétence seule de l'État moderne est insuffisante "pour combiner la palette des facteurs humains, industriel,

39. Taillemite, 2004. 
technologique, de commerce et de gestion qu'il fallait pour construire et soutenir une marine de guerre ${ }^{40} »$. Nicolas Rodger insiste sur le fait que les Nations où la politique se fonde sur une large coalition d'intérêts sont bien mieux armées pour devenir des puissances maritimes, autrement dit, le caractère social est décisif. Une « société souple » est indispensable, l'argent seul ne suffit pas, "les gouvernements absolutistes ne parvenaient pas à susciter la puissance maritime parce qu'ils avaient du mal à rassembler les grandes coalitions sociales nécessaires pour une entreprise si complexe". Si la puissance maritime atteint le degré plus élevé dans " des sociétés et des systèmes politiques ouverts et souples " c'est qu'elles favorisent le commerce et l'industrie, les armées de ces États étant alors considérées comme l'image de la société à laquelle ils aspirent.

Aujourd'hui, le concept global d'action de l'État en mer inclut la sécurité et la sûreté des activités en mer. La gouvernance des affaires de la mer renvoie au dispositif d'orientation des activités maritimes et à la définition de l'arbitrage entre des activités concurrentes, et par là aux conditions du développement d'une économie maritime. Les intérêts des États et des acteurs doivent aussi être convergents.

Il n'est pas très aisé de trouver dans la littérature du XVIII ${ }^{\mathrm{e}}$ siècle une définition de ce que devait être la politique maritime du royaume dans une approche globale. C'est d'abord l'histoire des réussites commerciales des États qui a retenu l'attention et donné lieu à des analyses. Il existe bien sûr des mémoires sur la stratégie navale de la France ou sur le commerce maritime dans les fonds d'archives, mais l'inventaire n'en est pas fait, de même, qu'il n'existe pas de corpus de textes pouvant éclairer la question de la politique globale au XvIII ${ }^{\mathrm{e}}$ siècle. L'étude de l'argumentation utilisée dans la production législative serait certainement instructive, notamment en comparant celle de l'époque Colbert-Seignelay à celle des deux décennies précédant la Révolution française. Quand on prend en compte les différentes tâches et domaines qui incombent au secrétariat d'État de la Marine, la potentialité d'une action globale est évidente. Le système fut construit par Colbert et ses successeurs dans ce but. Le ministre est aussi membre du Département des intendants du Commerce et contrôle tout le commerce extérieur maritime. Les secteurs et espaces commerciaux ont été bien délimités, Europe, Amérique, Afrique, Indes orientales, Pêches et

40. Rodger, 2004. 
Méditerranée. Les almanachs royaux permettent d'en donner les termes exacts et de constater leur fixité jusqu'en $178^{41}$. L'Encyclopédie méthodique marine, si brillante dans son contenu, n'apporte pas d'éléments particuliers sur le sujet de la politique maritime globale, sauf en quelques articles où sont définis par exemple les ports ou la police de la navigation. Toutefois, le "Discours préliminaire », qui démarre par une sorte d'apologie du perfectionnement de la navigation, rappelle de manière succincte le rôle essentiel de la Marine comme étant " un objet considérable dans certains États » et au surplus, une branche si importante du gouvernement qui intéresse " toute la nation ${ }^{42}$ ". Le " tableau analytique " ou "système encyclopédique de Marin " permet d'ailleurs de voir comment les rédacteurs des trois volumes de l'Encyclopédie méthodique ont construit l'arborescence des connaissances sur le sujet. La branche "Constitution et régime de la Marine » a pour base les ordonnances « de nos rois ». Les ordonnances qui concernent la marine marchande sont la base des jugements de la juridiction de l'Amirauté. Le sentiment qui prévaut à la lecture de ces quelques lignes, est celui, au fond, de deux mondes parallèles, qui se rencontrent par la captation des équipages pour la guerre. Malgré la très grande qualité des articles de la Méthodique, on y trouve donc essentiellement des informations normatives, techniques et savantes sur la marine de guerre.

Renouer avec l'espace territorial littoral par le point de vue d'en haut, c'est mettre la politique et l'État au cœur des articulations de la France maritime. Les travaux des dernières décennies ont bien montré le rôle de l'État dans ses aspects plutôt coercitifs et réglementaires, faisant du littoral, ce " territoire du vide ", paradoxalement, une zone sous surveillance, surencadrée, voire surcontrôlée à l'époque moderne ${ }^{43}$. C'est aussi comprendre l'action politique sous l'Ancien Régime, au sens de la mise en œuvre d'un programme, au sens de concevoir et d'ajuster une politique maritime à un contexte et des ambitions. Il apparaît dans la production historiographique française pour l'époque moderne que cette définition répond toujours au grand dessein de Colbert, précédé de celui de Richelieu. Au $\mathrm{XVIII}^{\mathrm{e}}$ siècle, les ministres de la Marine, ont, semble-t-il, perdu leur vue d'ensemble, ils paraissent ne s'occuper que de la guerre. Peu d'ouvrages sur l'histoire maritime s'intéressent à eux, leurs actes en matière d'organisation

41. Llinares et Ulbert (dir.), 2017.

42. Encyclopédie méthodique marine, 1783-1787, "Discours préliminaire ".

43. Le Bouëdec et Chappé (dir.), 2000. 
navale retiennent généralement l'attention dès lors qu'il s'agit d'évaluer les résultats et de fustiger les faiblesses de l'organisation structurelle qui échappaient souvent aux militaires et une stratégie hésitante.

L'on peut aussi juger de l'action politique et de ceux qui en ont la responsabilité à travers leur capacité à communiquer et à promouvoir leurs idées. La décennie qui encadre la guerre d'Indépendance américaine est sans doute une étape essentielle, même si le secrétariat d'État de la Marine n'a pas véritablement réussi à créer un journal d'information largement diffusé et susceptible d'embrasser toutes les questions importantes de la vie maritime, qu'il s'agisse de la navigation, du commerce, de la pêche, de la marine de guerre, de la législation, des sciences ou des colonies. Il a laissé à d'autres le soin de construire cette information, sauf à produire un almanach classique ou annuaire de la Marine du roi $^{44}$. La diffusion d'une information maritime existe, mais elle demeure encore trop fragmentaire car éparpillée dans les gazettes, ou trop spécialisée et cantonnée dans les ports marchands. De ce point de vue, le contexte de guerre navale est extrêmement porteur, tout concourt à faire éditer un vrai journal spécialisé en France. Le ministre Antoine de Sartine et les premiers commis des bureaux à Versailles, comprennent la nécessité d'une information globale qui serve les intérêts maritimes du royaume. De fait, le Journal de Marine proposé en 1776, depuis Brest, par Etienne Blondeau, professeur de mathématiques et membre de l'Académie de Marine, est approuvé et parrainé par le ministre. Publié pendant la guerre à partir de 1778 , le journal a ébauché un projet global et utilitaire sans pouvoir répondre à toutes les attentes des lecteurs ${ }^{45}$. On remarque ainsi que l'intégration de l'espace littoral à l'économie et à l'action politique par l'information se fait de manière très fragmentaire, à partir de matériaux et de réseaux que l'État royal ne contrôle pas.

Comme l'a souligné Alain Cabantous, le destin maritime de la France s'exprime véritablement à partir des années 1830 dans une littérature

44. L'État de la Marine est publié à Paris chez Nyon en format in- $24^{\circ}$, puis chez d'Houry à partir de 1784 en format in- $18^{\circ}$ : "sur le titre, petite vignette représentant une ancre sur un rocher, au fond, dans le lointain, un vaisseau. Au-dessus, dans les nuages, l'écusson fleurdelisé »; « l'État de la Marine, intéressant surtout pour les renseignements qu'il fournit sur certaines familles de l'ancienne France sera continué par L'État général de la Marine ». L'almanach des colonies y est ajouté à partir de 1778. Les Etrennes maritimes, pour l'année 1762, contenant des idées générales de la Marine et des vaisseaux et l'état des officiers de la Marine à la fin de 1761. À Paris, chez Nyon, quai des Augustins, 1761 , in- $24^{\circ}$.

45. Llinares, 2013. 
romanesque, poétique, technique et dans l'existence de revues spécialisées de « la chose maritime ». Intérêts multiples sur tout ce qui touche à la mer et support écrits divers caractérisent la production de cette période romantique destinée à un lectorat qui dépasse désormais les seuls professionnels ou les simples curieux. La construction d'une «identité maritime " française serait donc l'œuvre parfaitement réussie du mitan du XIX ${ }^{e}$ siècle $^{46}$.

\section{S'approprier la mer, maritimité et maritimisation : un essai de réflexion collective}

La prise de conscience que ces tendances récentes en historiographie sont pertinentes pour une étude comparée de l'histoire maritime de la Russie et de la France a donné lieu à l'organisation de deux séminaires internationaux russo-français, dont plusieurs contributions sont réunies dans ce dossier. L'idée des séminaires a pris corps à la fin de 2017, lorsqu'Alexei Kraikovski a séjourné à Paris en qualité de professeur invité de l'École normale supérieure (LABEX TransfertS). Les discussions qui ont suivi ses interventions consacrées aux divers aspects de l'histoire maritime russe du $\mathrm{XVIII}^{\mathrm{e}}$ siècle ont mis en évidence de nombreux parallèles entre ses propres recherches et les problématiques de collègues français. Grâce au soutien actif d'Irina Gouzévitch et de Liliane Hilaire-Pérez, un séminaire a été organisé dans les délais record et a suscité un grand intérêt chez les historiens maritimistes français. Le séminaire tenu le 22 décembre 2017 à l'Université Paris-Diderot a réuni onze communications introduites par l'exposé panoramique de Sylviane Llinares offrant un vaste aperçu de l'état des études maritimes en France.

Le succès du premier séminaire a donné une impulsion à l'organisation d'une seconde rencontre tenue cette fois-ci à l'Université nationale-École supérieure d'Économie (HSE) de Saint-Pétersbourg, les 18-21 mai 2019. Ce séminaire a attiré, outre les participants russes et français, des collègues du Royaume-Uni. La palette thématique du séminaire a également été élargie, le programme comprenant une discussion sur les possibilités

46. Cabantous, 2005. 
d'une représentation publique de l'histoire maritime en termes de Public History.

Les séminaires ont permis de confronter les approches, de proposer un premier bilan du travail collectif, de discuter de la genèse du concept de maritimité dans les études dédiées à l'histoire maritime de la France et de réfléchir aux applications possibles de cet outil de recherche à l'exploration des sources russes, avec une perspective de mise en comparaison des expériences maritimes française et russe.

On a pu également s'appuyer sur ces préalables théoriques et collaboratifs pour définir collectivement un certain nombre de domaines cruciaux de recherche où la comparaison des expériences historiques russe et française est pertinente. Tout d'abord, il existe, dans les deux cas, des régions « critiques " dont l'histoire revêt une importance particulière pour la formation de l'idée nationale de la maritimité. Pour la France, ce sont les zones clés de la côte atlantique et méditerranéenne, et pour la Russie, l'Arctique et la Baltique. En effet, c'est dans les régions du Nord et du Nord-ouest que s'est forgée l'expérience russe aux multiples facettes de l'interaction avec l'élément marin au Moyen Âge et au début de l'époque moderne. Il ne s'agit pas seulement du déploiement d'une tradition nationale de construction navale et de navigation, mais surtout de pratiques maritimes dans la vie sociale et quotidienne à différents niveaux, ce qui peut être qualifié de maritimité ${ }^{47}$. Comme l'a montré l'expérience récente de l'étude collective dédiée à l'histoire de l'artisanat russe du XviII ${ }^{\mathrm{e}}$ siècle au Svalbard (Spitzbergen), l'approche basée sur l'idée de maritimité offre une vaste perspective de recherche en stimulant de nouveaux questionnements et en attirant des ressources jusqu'alors inexploitées car considérées comme peu pertinentes dans le cadre des approches héritées ${ }^{48}$.

On observe également, dans les deux cas, que les dimensions multiples de la notion de maritimité conduisent à prendre en compte la diversité des voies par lesquelles celle-ci pénètre dans la société, rendant le pays maritime. On notera également qu'il est assez difficile d'établir une hiérarchie justifiée de ces voies, d'indiquer que certaines d'entre elles sont plus importantes que d'autres. La maritimité apparaît comme le résultat d'interactions sociales plurielles et complexes.

47. Kraikovski, 2015.

48. Dadykina, Kraikovski et Lajus, 2017. 
Enfin, en France comme en Russie, on constate l'évidence du rôle central de la connaissance de la mer au sens large. La maritimité repose, avant tout, sur une idée claire des propriétés de la mer en tant qu'objet naturel. Notons encore qu'il ne s'agit pas seulement d'une connaissance scientifique objective au sens moderne du terme, mais aussi d'un « imaginaire scientifique » qui a pu être ajusté dans le processus d'amélioration des méthodes scientifiques. Deuxièmement, la maritimité signifie la maîtrise d'un certain ensemble de connaissances pratiques qui permettent de reconstituer efficacement l'interaction de la société avec la mer. Soulignons que le concept de maritimité est indissociable d'une vision extensive de cet ensemble de connaissances. Encore une fois, il ne s'agit pas uniquement des compétences nécessaires pour construire un navire et pour se déplacer en mer, mais d'un complexe de connaissances et de savoir-faire dont la mise en œuvre conduit à l'émergence et à la consolidation des grands systèmes techniques, faisant de la mer un élément routinier de la vie quotidienne, comme cela s'est produit dès l'origine à Saint-Pétersbourg ${ }^{49}$.

Enfin, la maritimité renvoie à une certaine souplesse, à une variabilité et une durabilité dans l'utilisation des infrastructures marines, rendue possible grâce aux ressources de la mémoire, de l'histoire et de la construction des identités. Une société maritime ne considère pas les objets matériels, les pratiques sociales et individuelles d'interaction avec la mer uniquement du point de vue de leur utilité, elle continue à les intégrer même après que leur fonction originelle a été complétement perdue. Ces objets et pratiques sont au cour des processus de construction identitaire à travers la patrimonialisation (heritagization), la commémoration et, finalement, la représentation que la communauté projette d'elle-même vers l'extérieur ${ }^{50}$.

Les articles groupés dans ce dossier confirment ces observations et les développent. Les contributions permettent, d'une part, d'interroger l'aspect spatial de la maritimité. Les auteurs russes optent pour une géographie clairement délimitée en mettant au centre de leur recherche une région particulière, même si très étendue. Pour Julia Lajus, Pavel Filin, Margarita Dadykina et Anastassia Bogomazova, il s'agit du Nord russe, tandis que Pavel Demchenko se positionne comme historien de la Baltique. Les

49. Kraikovski et Lajus, 2019.

50. Kraikovski, Bogachev and Lomakina, 2020. 
collègues français abordent la perspective géographique différemment, en focalisant leurs recherches sur les problématiques transrégionales.

En même temps, les contributions des chercheurs français s'organisent autour des problématiques liées à la connaissance de la mer au sens le plus large. Romain Grancher et Bruno Marnot s'intéressent au développement des techniques qui déterminent les modalités de l'interaction de la société avec la mer. Si dans le premier cas, l'auteur s'attache à réfléchir à l'articulation entre le progrès technique et l'exploitation des ressources naturelles, dans le second cas le développement des techniques est pensé comme la construction de grands systèmes techniques conditionnant à long terme le fonctionnement des entreprises logistiques.

D'une certaine manière, l'article de David Plouviez fait écho à ces travaux, l'auteur considérant de plus, la logistique portuaire comme un champ d'interaction des acteurs. Le projecteur est mis ici sur les constructeurs de navires dans un long XVIII ${ }^{\mathrm{e}}$ siècle, porteurs d'une expertise spécifique dont l'émergence a eu des conséquences sur les rapports de pouvoirs dans l'environnement portuaire. L'étude s'inscrit dans le sillage de l'important renouvellement de l'histoire des trafics maritimes et des élites négociantes portuaires débuté à partir des années 1960, tout en s'ouvrant vers l'étude les sociétés littorales, articulée autour des concepts d'identités maritimes et de pluriactivités, avec la volonté de saisir ces populations dans leur diversité socioprofessionnelle. Pavel Demchenko adopte la même approche pour explorer les interactions entre les parties prenantes dans les ports de la Baltique. Anastassia Bogomazova et Margarita Dadykina proposent l'étude du développement de la maritimité russe au prisme de l'économie monastique. Loin d'établir une hiérarchie des modalités d'interaction entre la communauté monastique et la mer, les chercheuses privilégient une image intégrée et cohérente de la relation entre la conception du monde des moines et les principes généraux de leur vie, d'une part, et la mise en œuvre de techniques et de pratiques d'utilisation de la mer, d'autre part.

Julia Lajus explore d'autres aspects particuliers de la maritimité. Mettant l'accent sur la matérialité des connaissances, elle analyse le lien entre l'acquisition et la maitrise de certains instruments scientifiques et la perception évolutive de la mer en tant qu'objet naturel. Elle attire aussi l'attention sur la question du genre, soulignant la détention de connaissances par les femmes, non sans questionner le caractère masculin de la maritimité 
comme construction culturelle. Ces observations sont en phase avec les études de Carlotta Andrews qui a, pour sa part, mis en évidence les changements survenus en matière de genre de la maritimité.

Les contributions d'Irina Gouzévitch et de Dmitri Gouzévitch occupent une place à part dans notre collection de textes. Cela tient à la dimension géographique de leur étude. L'article de Dmitri Gouzévitch étudie le voyage de Pierre ${ }^{\text {er }}$ dans les ports de la Baltique, de la mer du Nord et de La Manche en 1717 et interroge cette tournée du littoral européen à travers ses expériences maritimes antérieures d'une part, et les perspectives d'exploitation plurielle du golfe de Finlande d'autre part. Irina Gouzévitch consacre son texte à un épisode crucial de la cartographie du Pacifique Nord par les membres de la Deuxième expédition du Kamchatka (17331743) qui a permis de grandes découvertes en s'appuyant sur des « objets géographiques fantômes ". Ces deux articles interrogent, chacun à sa manière, le phénomène de la mer imaginaire, en démontrant le lien entre la maritimisation de la société et la place prise par les étendues marines dans les représentations du monde.

Enfin, le thème de l'exploitation à long terme des infrastructures maritimes en lien avec la construction des identités maritimes est discuté dans les articles de Géraldine Barron, Sylvain Laubé et Pavel Filin. Curieusement, la perspective offerte par les études sur la maritimité s'est avérée suffisamment large et flexible pour permettre aux trois chercheurs, tout en restant dans le cadre d'un paradigme unique, de proposer chacun une vision originale du patrimoine maritime et de ses représentations. Là où Géraldine Barron analyse la représentation de la maritimité au XIX ${ }^{\mathrm{e}}$ siècle comme transformant cette notion en un objet de représentation d'une sorte d'artefact du passé, Sylvain Laubé y voit un aspect majeur dans l'interprétation de la patrimonialisation des infrastructures techniques et industrielles. Il s'attache, notamment, à mieux articuler la notion de la maritimité avec les concepts de "paysages culturels maritimes » et de " lieux de savoirs » et à la rendre opérante grâce au couplage avec le méta-modèle "Any-artefact ", développé à Brest depuis 2012 dans le cadre de travaux de recherche interdisciplinaires en histoire des techniques et humanités numériques. Quant à Pavel Filin, il évoque les problèmes urgents de la politique contemporaine de construction de la mémoire en Russie, avec son regard particulier sur l'Arctique en tant que région essentielle pour comprendre le passé national. 
Nos deux séminaires se sont avérés extrêmement fertiles pour l'animation des recherches franco-russes en matière d'histoire et de patrimoine maritimes. Les collaborations conclues dans ce cadre ont débouché sur la décision d'associer les chercheurs russes dans une série de projets internationaux pilotés en France par Sylviane Llinares et Romain Grancher dans le cadre du GIS Histoire et Science de la Mer et par Liliane Hilaire-Pérez, dans le cadre du laboratoire Identités-Cultures-Territoires (Université de Paris) et du GDR 2092 "Techniques et production dans l'histoire ". Les séminaires ont reçu le soutien de l'Université de Paris (alors intitulée Paris Diderot), de la Commission scientifique de l'Université nationale de Recherche "École supérieure d'Économie " (UNR ESE), antenne de Saint-Pétersbourg, du Centre de formation des administrateurs de l'UNR ESE Centre Kochubej. Nous tenons à exprimer notre profonde reconnaissance à toutes ces institutions.

\section{Bibliographie}

AkCan Esra, Architecture in Translation: Germany, Turkey, and the Modern House, Duke University Press, Durham NC, 2012.

Alegret Joan Lluís, Carbonell Camós Eliseu (dir.), Revisiting the Coast: New Practices in Maritime Heritage, Institut Català de Recerca en Patrimoni Cultural, Girona, 2014.

Alpers Edward A., The Indian Ocean in World History, Oxford University Press, Oxford, 2014.

Andrews Carlotta, "Community uses of maritime heritage in Bermuda : A heritage ethnography with museum implications ", thèse de doctorat, Université de Cambridge, 2010.

Armitage David, Bashford Alison (dir.), Pacific Histories: Ocean, Land, People, Macmillan International Higher Education, New York, 2014.

Braudel Fernand, La Méditerranée et le monde méditerranéen à l'époque de Philippe II, Armand Colin, Paris, 1966.

Buchet Christian, Une autre histoire des océans et des hommes, Laffont, Paris, 2004.

Buchet Christian, Balard Michel (dir.). The Sea in History. The Medieval World, Boydell \& Brewer, New York, 2017.

Buchet Christian, Le Bouëdec Gérard (dir.), The Sea in History. The Early Modern World, Boydell \& Brewer, New York, 2017. 
Buchet Christian, Rodger Nicholas (dir.), The Sea in History. The Modern World, Boydell \& Brewer, New York, 2017.

Buchet Christian, Arnaud Pascal, De Souza Philip (dir.), The Sea in History. The Ancient World, Boydell \& Brewer. New York, 2017.

Cabantous Alain, "Lectures culturelles", dans Cabantous Alain, Lespagnol André et PÉron Françoise (dir.), Les Français, la terre et la mer, XIII ${ }^{\circ}$-XX siècles, Fayard, Paris, 2005, p. 415-420.

Cabantous Alain, Lespagnol André et Péron Françoise (dir.), Les Français, la terre et la mer, XIII'-XX' siècles, Fayard, Paris, 2005.

Coll., La Maritimisation du Monde de la préhistoire à nos jours, GIS d'histoire maritime, PUPS, Paris, 2016.

Corbin Alain, Le Territoire du vide : l'Occident et le désir du rivage (1750-1840), Flammarion, Paris, 1990.

Dadykina Margarita M., Kraikovski Alexei, Lajus Julia, Primorskie promysly na Shpicbergene $v$ XVIII-nachale XIX v. Issledovanie. Dokumenty, Al'jans-Arheo, Saint-Pétersbourg, 2017.

DAY Ann, LunN Ken, "British maritime heritage : Carried along by the currents? ", International Journal of Heritage Studies, ${ }^{\circ}$ 9-4, 2003, p. 289-305.

DeLoughrey Elisabeth, "Submarine futures of the anthropocene ", Comparative Literature, $\mathrm{n}^{\circ}$ 69-1, 2017, p. 32-44.

Encyclopédie méthodique marine, Paris et Liège, Panckoucke, 3 vol, 1783-1787.

Eski Yarin, Policing, Port Security and Crime Control: An Ethnography of the Port Securityscape, Routledge, Londres, 2016

Fleisher Jeffrey et al., "When did the Swahili become maritime? ", American Anthropologist, $\mathrm{n}^{\circ}$ 117-1, 2015, p. 100-115.

François Luc, Isaacs Ann Katherine (dir.), The Sea in European History, Edizioni PLUS, Pise, 2001.

Grataloup Christian, Faut-il penser autrement l'histoire du monde?, Armand Colin, Paris, 2011.

Greene Jack P., Morgan Philip D. (dir.), Atlantic History: A Critical Appraisal, Oxford University Press, Oxford, 2009.

Harlaftis Gelina, "Maritime history or the History of Thalassa ", dans Harlaftis Gelina, Sbonias Kostas, Karapidakis Nikos, Vaiapoulos Vaios (dir.) New Ways of History: Developments in Historiography, I. B. Tauris, Londres, 2010, p. 211-229. 
Hilaire-Pérez Liliane, L’Expérience de la mer. Les Européens et les espaces maritimes au XVIII siècle, Seli Arslan, Paris, 1997.

Kraikovski Alexei, Bogachev Nikita, Lomakina Ivanna, "Playing maritime capital : The Baltic Sea in the touristic representations of St.-Petersburg ", International Journal of Maritime History, $\mathrm{n}^{\circ}$ 32-4, 2020, p. 928-945.

Kraikovski Alexei, Lajus, Julia, "The metropolitan bay: Spatial imaginary of imperial St.-Petersburg and maritime heritage of the Gulf of Finland ", Humanities, $\mathrm{n}^{\circ}$ 8-1, 2019, p. 1-12.

Kraikovski Alexei, "'The Sea on one side, trouble on the other' : Russian marine resource use before Peter the Great ", The Slavonic and East European Review, n 93-1, 2015, p. 39-65.

KROTOv Pavel, «Ambiguous relations between Russia and the sea, causes and consequences ", dans Buchet Christian, Le BouËDec Gérard (dir.), The Sea in History. The Early Modern World, vol. 3, Boydell\&Brewer, Londres, 2017, p. 509-526.

Laget de Podio (Le chev. de), Le Parfait capitaine, Librairie orientale de Prosper Dondey-Dupré, Paris, 1828, $2^{\mathrm{e}}$ éd., "Introduction », p. 1-4.

Laget de Podio (Le chev. de), Les Pêcheurs de la ville de Marseille et de sa banlieue, qui ont chargé $M$. Laget de Podio,... de demander en leur nom l'exécution des lois sur la police de la pêche, à M. le procureur du Roi près le tribunal civil de Marseille..., impr. de M. Olive, Marseille 1835.

LE BouËDEC Gérard (dir.), « La Recherche internationale en histoire maritime : essai d'évaluation ", Revue d'histoire maritime, nº10-11, 2010.

Le BouËDec Gérard, Chappé François (dir.), Pouvoirs et Littoraux du XV au XXe siècle, PUR, Rennes, 2000.

Le Corbeiller Clare. "China into Delft: A note on visual translation", The Metropolitan Museum of Art Bulletin, $\mathrm{n}^{\circ}$ 26-6, 1968, p. 269-276.

Leikin Julia, "Across the seven seas: Is Russian maritime history more that regional history ?", Kritika, n 17-3, 2016, p. 631-646.

Listr Antoine, L'Héritage des Lumières. Ambivalences de la modernité, Seuil, Paris, 2019.

LLINARES Sylviane, «L'information maritime pendant la guerre d'Amérique ou comment ne pas faire de politique dans le Journal de Marine (1776-1783) », dans Bourquin Laurent, Hamon Philippe, Hugon Alain et Lagadec Yann (dir.), La Politique par les armes. Conflits internationaux et politisation (XVe-XIX siècle), PUR, Rennes, 2013, p. 59-279.

Llinares Sylviane, "Le guide portuaire de Nicolas Ponce ou la mise à jour textuelle des vues des ports de France de Nicolas Ozanne (1776-1819) ", dans Llinares Sylviane (dir.), Avec vue sur la mer, Éd. du CTHQ, Paris, 2011, p. 70-83. 
Llinares Sylviane et Ulbert Jörg (dir.), La Liasse et la plume, les bureaux du secrétariat d'État de la Marine (1669-1792), PUR, Rennes, 2017.

Lorgeoux Jeanny et Trillard André, " Maritimisation : la France face à la nouvelle géopolitique des océans ", Rapport d'information au nom de la commission des Affaires étrangères, de la Défense et des Forces armées, nº 674, 17 juillet 2012.

Mercier Louis Sébastien, Le Tableau de Paris, tome 1, 1783, chapitre xxxiv, " Poissons de mer ", Slatkine, Genève, p. 60-61.

Miller Peter N. (dir.) The Sea: Thalassography and Historiography, University of Michigan Press, Ann Arbor, 2013.

North Michael, The Baltic: A History, Harvard University Press, Cambridge MA, 2015.

Péron Françoise et Rieucau Jean (dir.), La Maritimité aujourd'hui, L'Harmattan, Paris, 1996.

Pétré-Grenouilleau Olivier, Les Négoces maritimes français, XVII ${ }^{\top}$-XX siècles, Belin Sup. Histoire, Paris, 1997.

Robinson Sam, "Scientific imaginaries and science diplomacy: The case of ocean exploitation ", Centaurus, 2020, p. 1-21.

Rodger Nicholas A.M, "Une marine moderne dans une société moderne ? Le cas de la Grande-Bretagne ", dans Buchet Christian, Meyer Jean, Poussou Jean-Pierre (dir.), La Puissance maritime, PUPS, Paris, 2004, p. 545-552.

Taillemite Etienne, «La puissance maritime : une absente des mentalités françaises ", dans Buchet Christian, Meyer Jean, Poussou Jean-Pierre (dir.), La Puissance maritime, PUPS, Paris, 2004, p. 533-543.

Vergé-Franceschi Michel, Chronique maritime de la France d'Ancien Régime 1492-1792, SEDES, Paris, 1998.

Villain-Gandossi Christiane et Rieth Éric (dir.), Pour une histoire du fait maritime, CTHS, Paris, 2001.

Worthington David, "Introducing the new coastal history: Cultural and environmental perspectives from Scotland and beyond ", dans Worthington David (dir.) The New Coastal History. Cultural and Environmental Perspectives from Scotland and Beyond, Palgrave Macmillan, Londres, 2017, p 3-30. 
Sylviane Llinares, Alexei Kraikovski et Irina Gouzévitch

\section{Les auteurs}

Sylviane Llinares est professeure des universités en histoire moderne à l'Université Bretagne Sud, elle est directrice-adjointe du laboratoire TEMOS Temps Mondes Sociétés UMR 9016 CNRS et directrice du GIS Histoire \& Sciences de la Mer CNRS INSHS. Ses recherches portent sur l'histoire des cultures et des politiques maritimes à l'époque moderne.

Alexei Kraikovski a fait ses études supérieures en histoire à Saint-Pétersbourg. Depuis 2017, il est associate professor à l'Université nationale de recherche École supérieure d'Économie (HSE) de Saint-Pétersbourg (Laboratoire d'histoire écologique et technologique). Il est spécialiste d'histoire marine et côtière et d'histoire de la Russie du Nord aux XVII ${ }^{\mathrm{e}}-\mathrm{xVIII}$ e siècles. Il étudie en particulier l'histoire de la maritimité russe dans une perspective comparée.

Irina Gouzévitch est docteur en histoire des techniques (Université Paris 8), habilitée à diriger les recherches en histoire (Université de Paris), rattachée au Centre Maurice Halbwachs, CNRS-EHESS (Paris). Ses domaines de recherche sont les échanges interculturels Russie-Europe occidentale, les techniques et les empires, la circulation des connaissances scientifiques et techniques en Europe aux XVIII $x x^{e}$ siècles, l'enseignement technique, les réseaux de sociabilité, la mobilité des experts, les questions identitaires. 